\title{
Complete Genome Sequence of Porcine Circovirus 2b Strain Shandong
}

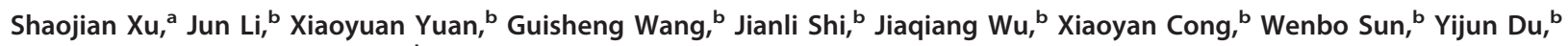 \\ Tongjie Chai, ${ }^{a}$ and Jinbao Wang ${ }^{b}$ \\ Department of Preventive Veterinary Medicine, College of Veterinary Medicine, Shandong Agricultural University, Taian, China, and Shandong Key Laboratory of Animal \\ Disease Control and Breeding, Institute of Animal Science and Veterinary Medicine, Shandong Academy of Agricultural Sciences, Jinan, China ${ }^{\mathrm{b}}$
}

Shandong is a porcine circovirus $2 \mathrm{~b}$ (PCV2b) strain that was isolated and purified from tissue samples from pigs with postweaning multisystemic wasting syndrome (PMWS) in the Shandong Province of China. Here, we report the complete genome sequence of strain Shandong, which may aid in understanding the molecular characteristics of this strain.

P orcine circovirus type 2 (PCV2) is the primary causative agent of porcine circovirus-associated diseases (PCVAD) in pigs. PCV2 has two major open reading frames (ORFs), ORF1 and ORF2. Studies of PCV2 genomic sequences show that the viruses can be further divided into distinct subtypes, also known as genotypes $(2,5)$. PCV2 genotype definition and nomenclature have been proposed $(4,6)$, with three genotypes identified to date $(6)$ including genotypes a (PCV2a) and b (PCV2b), which are the two main phylogenetic groups, and a third genotype, c (PCV2c), which has been found only in Denmark as yet (3). The signature motif in ORF2 can be used to distinguish PCV2a and 2b genotypes (1). For PCV2b viruses, the motif is $1479-1468$ TCA/AAC/CCC/ CG(T)C [amino acid sequence SNPR(L)]. For the PCV2a viruses, the motif is 1480-1469 ACC/AAC/AAA/ATC (amino acid sequence TNKI).

A PCV2 strain, designated Shandong, was isolated from tissue samples from pigs with postweaning multisystemic wasting syndrome (PMWS) in 2005 in the Shandong Province of China. The full-length genome of the isolated strain was amplified by PCR. The PCR product was purified with the QIAquick gel extraction kit (QIAquick) and cloned using the pMD18-T vector (TaKaRa) according to the manufacturer's instructions. Three positive clones for each fragment were sequenced by Sangon Biotech (Shanghai) Co., Ltd., using M13 universal forward and reverse sequencing primers. Genomic analysis was conducted using DNASTAR software (DNASTAR Inc.).

The result showed that the full-length genome of the PCV2 strain Shandong consists of 1,767 nucleotides (nt). The 14791468 motif of this strain is TCA/AAC/CCC/CTC (amino acid sequence SNPL), which is characteristic of PCV2b. This indicates that the strain belongs to the PCV2b genotype.

The results of sequence analysis showed that there were 11 ORFs in the PCV2b Shandong strain. The ORF2 nucleotide sequence of this strain is $705 \mathrm{nt}$, encoding 234 amino acids. Compared with eight other strains (HM027579, HM027580,
HM142894, HM142895, HM142896, HM142899, HM161710, and HM161711) of the PCV2b genotype obtained by our lab in 2010 , they had the same changes in six amino acid positions (at $68,90,121,134,169$, and 215 amino acids).

Nucleotide sequence accession number. The complete genome sequence of PCV2b strain Shandong has been deposited in GenBank and assigned accession no. DQ346683.

\section{ACKNOWLEDGMENTS}

The study was partly supported by the National Natural Science Funds (31201889, 31170146) and the Natural Fund of Shandong (ZR2010CQ003, ZR2010CQ044).

\section{REFERENCES}

1. Cheung A, et al. 2007. Detection of two porcine circovirus type 2 genotypic groups in United States swine herds. Arch. Virol. 152:1035-1044.

2. de Boisséson C, et al. 2004. Molecular characterization of porcine circovirus type 2 isolates from post-weaning multisystemic wasting syndromeaffected and non-affected pigs. J. Gen. Virol. 85:293-304.

3. Dupont K, Nielsen E, Baekbo P, Larsen L. 2008. Genomic analysis of PCV2 isolates from Danish archives and a current PMWS case-control study supports a shift in genotypes with time. Vet. Microbiol. 128:56-64.

4. Grau-Roma L, et al. 2008. A proposal on porcine circovirus type 2 (PCV2) genotype definition and their relation with postweaning multisystemic wasting syndrome (PMWS) occurrence. Vet. Microbiol. 128:23-35.

5. Olvera A, Cortey M, Segales J. 2007. Molecular evolution of porcine circovirus type 2 genomes: phylogeny and clonality. Virology 357:175-185.

6. Segalés J, et al. 2008. PCV-2 genotype definition and nomenclature. Vet. Rec. 162:867-868.

Received 30 September 2012 Accepted 1 October 2012 Address correspondence to Jinbao Wang, wangjb@saas.ac.cn, or Tongjie Chai, chaitj117@163.com

Shaojian Xu, Jun Li, and Xiaoyuan Yuan contributed equally to this work. Copyright $\odot$ 2012, American Society for Microbiology. All Rights Reserved. doi:10.1128/JVI.02739-12 